\begin{tabular}{ll|l} 
Case Reports in & \multicolumn{2}{c}{ Case Rep Gastroenterol 2014;8:270-275 } \\
\cline { 2 - 3 } Gastroenterology & $\begin{array}{l}\text { DOI: 10.1159/000368076 } \\
\text { Publisned onine: September 10, 2014 }\end{array}$ & $\begin{array}{l}\text { ○ 2014 S. Karger AG, Basel } \\
\text { www.karger.com/crg }\end{array}$ \\
\hline & $\begin{array}{l}\text { This is an Open Access article licensed under the terms of the Creative Commons } \\
\text { Attribution-NonCommercial 3.0 Unported license (CC BY-NC) (www.karger.com/OA- } \\
\text { license), applicable to the online version of the article only. Distribution permitted for non- } \\
\text { commercial purposes only. }\end{array}$
\end{tabular}
commercial purposes only.

\title{
A Case of Early Gastric Cancer Arising from Gastritis Cystica Profunda Treated by Endoscopic Submucosal Dissection
}

\author{
Naotaka Ogasawara ${ }^{a}$ Hisatsugu Noda $^{a}$ Yoshihiro Kondo ${ }^{a}$ \\ Takashi Yoshimine $^{a}$ Tomoya Sugiyama $^{\text {a }}$ Mikitoshi Kimura $^{a}$ \\ Satoshi Inoue ${ }^{a}$ Emiko Takahashi $^{\mathrm{b}}$ Makoto Sasaki $^{\mathrm{a}}$ Kunio Kasugai ${ }^{\mathrm{a}}$ \\ Departments of a Gastroenterology and ${ }^{b}$ Pathology, Aichi Medical University School of \\ Medicine, Nagakute, Japan
}

\section{Key Words}

Endoscopic submucosal dissection · Endoscopic ultrasonography · Gastric cancer · Gastritis cystica profunda. Submucosal tumor

\begin{abstract}
Gastritis cystica profunda (GCP) consists of hyperplasia and cystic dilatation of the gastric glands extending into the submucosa. It occurs in the residual stomach post surgery and in the unoperated stomach. GCP is considered a benign lesion, but there is controversy about its malignant potential. We report a case of early gastric cancer arising from GCP treated by endoscopic submucosal dissection (ESD) in a 55-year-old unoperated man. Upper gastrointestinal endoscopy revealed a 15-mm diameter submucosal tumor (SMT) in the upper corpus of the stomach. The surface had angiotelectasia and slight depression covered with normal mucosa. Neither ulceration nor erosion was seen. Narrow-band imaging endoscopy showed no abnormalities suggesting gastric cancer. Endoscopic ultrasonography visualized the internally low-echoic SMT, harboring tiny cystic lesions, mainly within the second and third layers of the gastric wall. The SMT was removed by ESD to avoid retention and allow for comprehensive diagnosis. It was diagnosed as GCP with partial well-differentiated adenocarcinoma without involvement of the lateral and deep margins, lymphatic invasion, vascular invasion and perineural invasion. The gastric epithelium comprised normal mucosa without dysplasia. ESD seems to be useful for the diagnosis of SMT, including GCP harboring gastric cancer, and avoids unnecessary surgical procedures.

(C) 2014 S. Karger AG, Basel
\end{abstract}

Naotaka Ogasawara, MD

Department of Gastroenterology

Aichi Medical University School of Medicine

1-1 Yazakokarimata, Nagakute, Aichi 480-1195 (Japan)

E-Mail nogasa@aichi-med-u.ac.jp 
Ogasawara et al.: A Case of Early Gastric Cancer Arising from Gastritis Cystica Profunda Treated by Endoscopic Submucosal Dissection

\section{Introduction}

Gastritis cystica profunda (GCP) histologically comprises hyperplasia and cystic dilatation of the gastric glands extending into the submucosal layer $[1,2]$. Since the majority of GCP cases are secondary changes caused by prolonged chronic inflammation, ischemia, gastric surgery or suturing material, injury of the muscularis mucosae is assumed to trigger the ectopic entrapment of gastric glands in the submucosa [1,2]. Macroscopically, GCP often presents as a submucosal tumor (SMT), solitary diffuse polyps or a giant gastric mucosal fold [3-5]. As specimens obtained with endoscopic forceps biopsy are usually limited to the mucosa, histopathological information of the submucosa is not available when GCP presents as SMT in particular. It is also difficult to precisely diagnose GCP presenting as SMT using endoscopic ultrasonography (EUS). Therefore, surgical treatment may be required for a precise diagnosis of GCP, with features mimicking SMT. GCP is usually regarded as a benign lesion $[2,6,7]$, but there are some controversies about its malignant potential. Recently, there have been some reports of GCP accompanied by gastric carcinoma or adenoma with high-grade dysplasia. Therefore, GCP is thought to be a precancerous lesion.

Herein, we report a case of early gastric cancer arising from GCP presenting SMT features treated by endoscopic submucosal dissection (ESD) in an unoperated patient. ESD is useful for the diagnosis of such cases because GCP mimicking SMT might not be precisely diagnosed by endoscopic forceps biopsy and EUS, and because SMT might harbor a precancerous lesion.

\section{Case Report}

A routine health screen using upper gastrointestinal endoscopy revealed a SMT in a 55-year-old apparently healthy man with no known family history of gastrointestinal disorders or malignant diseases. He had no abdominal discomfort and no stomach or intestinal symptoms. Physical findings were unremarkable and initial biochemical and hematological parameters were within the normal limits. Endoscopy (GIF-H260Z; Olympus, Tokyo, Japan) indicated a 15-mm diameter SMT with normal overlying mucosa in the giant curvature of the upper corpus of the stomach (fig. 1a, b). The surface of the SMT had angiotelectasia and slight depression and was covered with normal mucosa. However, neither ulceration nor erosion was seen. Narrow-band imaging endoscopy showed no abnormal finding suggesting gastric cancer (fig. 1c). The mucosa of the whole stomach was normal, without chronic gastritis. Mucosal biopsies of both the middle portion and antrum of the stomach confirmed the absence of Helicobacter pylori infection. Tests for H. pylori immunoglobulin G levels using blood examination were also negative. Computed tomography detected no SMTs or abnormal findings in any other organ and no swollen lymph nodes. EUS visualized the SMT mainly within the second and third layers of the gastric wall (fig. 1d). The tumor was internally lowechoic and harbored tiny cystic lesions (fig. 1d). The endoscopy and EUS findings indicated that the SMT was localized in the submucosal propria; however, it was too small to perform fine needle aspiration (FNA) biopsy under EUS guidance. A biopsy specimen obtained from the SMT did not include the tumor contents and therefore a definitive pathological diagnosis of the tumor could not be achieved. However, a precise diagnosis was required to rule out malignancy. The patient refused to undergo surgery, but consented to undergo endoscopic treatment. To completely resect the SMT using only endoscopic mucosal resection was considered very difficult. Therefore, the SMT was resected using ESD and not endoscopic mucosal resection to avoid SMT retention and to comprehensively diagnose the SMT. The 
Ogasawara et al.: A Case of Early Gastric Cancer Arising from Gastritis Cystica Profunda Treated by Endoscopic Submucosal Dissection

resected specimen was $35 \times 20 \mathrm{~mm}$ and the tumor was $12 \times 12 \mathrm{~mm}$ (fig. 2a). Histologically, cystic dilatation of the gastric glands extending into the submucosal layer was present, and the SMT was diagnosed as GCP (fig. 2b, c). The gastric epithelium comprised normal mucosa without any dysplasia (fig. 2b, c). Well-differentiated adenocarcinoma was partially seen within the GCP (fig. 2c, d). However, there was no involvement of the lateral and deep margins, lymphatic invasion, vascular invasion or perineural invasion. Therefore, the histopathological findings concurred with a diagnosis of early gastric cancer (SM2, 1,000 $\mu \mathrm{m}$ ) arising from GCP. The patient refused further surgical treatment and was subsequently followed up as an outpatient. He has remained free of recurrence and metastasis or other clinical symptoms for 39 months.

\section{Discussion}

Since Littler and Gleibermann initially reported GCP in 1972 [8], numerous cases have been reported in the literature $[2,6,9,10]$. Diverse terminology for GCP has been used: diffuse submucosal cysts of the stomach [6], submucosal heterotopic gastric glands [7], gastritis cystica polyposa [8] or diffuse heterotopic cystic malformation of the stomach. GCP is found mainly in the posterior and anterior wall of the gastric body and in the intermediate zone between the fundic and pyloric glands. The mechanisms of GCP have been considered to be chronic inflammation, ischemia and presence of a foreign body [2, 6, 8-10]. Interruption of the muscularis mucosa may be caused by chronic inflammation of the gastric mucosa and ischemia, or by the effects of surgery and presence of suture material, resulting in migration of epithelial cells into the submucosal layer. This finally results in cystic dilatation of glands in the basal mucosa and submucosa, with superficial inflammation in the lamina propria. GCP has frequently been reported in the setting of prior gastric surgery, presumably because of mucosal prolapse or reflux of intestinal contents including bile juice $[2,6,8]$. However, it has been found not only at the residual stomach after gastrostomy, but also in the stomach of patients without any previous surgical treatment. Although GCP is also considered to be caused by chronic gastritis frequently associated with $H$. pylori infection, it remains uncertain whether GCP is associated with the early stage of its inflammatory process. In this case, the patient had no previous history of surgery or H. pylori infection, confirmed by histopathological examination and blood examination. Endoscopy indicated no inflammation of the gastric mucosa.

Although GCP is generally benign, there have been some reports of GCP associated with cancer $[1,2,11]$. Therefore, GCP has recently been considered a precancerous lesion. Experiments have shown that animals predisposed to $H$. pylori infection develop not only secondary GCP but also subsequent gastric carcinoma [12]. This close association between GCP and malignancy has been interpreted as concurrent sharing of causative factors common to both disease conditions [2,12]. According to the report by Iwanaga et al. [6], GCP accompanied $3.0 \%$ of gastric carcinoma cases. However, it is very difficult to make an early diagnosis of the cancer developed within the submucosal gland of GCP using endoscopy or tissue obtained from forceps biopsy.

Small SMTs are usually asymptomatic and incidentally detected during endoscopic or radiological examinations. One retrospective study has suggested that the incidence of gastric SMT is $0.36 \%$ [13]. SMTs are very difficult to accurately diagnose by endoscopic or radiological means. The differential diagnoses of SMTs include gastrointestinal stromal tumor, fibromatosis, inflammatory myofibroblastic tumors, neuroendocrine cell tumors, schwannomas, heterotopic pancreas, lipomas, cystic lesions, lymphomas and leiomyomas. 
Ogasawara et al.: A Case of Early Gastric Cancer Arising from Gastritis Cystica Profunda Treated by Endoscopic Submucosal Dissection

GCP includes SMTs that are endoscopically difficult to differentiate from other SMTs such as those described above, especially when they are small and have endoscopically non-specific findings. Although EUS and EUS-guided FNA are considered useful for diagnosing SMTs, these modalities cannot perfectly diagnose whole SMTs when EUS findings are non-specific or when SMTs are too small to be treated by FNA. In this case, the SMT was located in the second and third layers of the gastric wall and had the same homogeneous, low-echoic features. These findings excluded gastrointestinal stromal tumor, leiomyoma, schwannoma and lipoma from the differential diagnosis, but a more precise diagnosis by EUS remained impossible. On EUS, GCP is reported to consist of multiple cystic lesions within a thickened submucosal layer [14]. The EUS finding in our case showed tiny cystic lesions in the SMT presuming possible findings for GCP. However, the SMT was approximately $15 \mathrm{~mm}$ in diameter, which was considered too small to diagnose using EUS-guided FNA. Since endoscopy and EUS could not provide a precise diagnosis, the SMT was resected by ESD and finally diagnosed as GCP accompanied by partial well-differentiated adenocarcinoma. ESD appears to be useful for the diagnosis of SMTs including GCP, which may possibly harbor gastric cancer; in addition, many unnecessary surgical procedures can be avoided in such cases of undiagnosed SMTs.

\section{Disclosure Statement}

The authors have no conflict of interest associated with this paper.

\section{References}

1 Mitomi H, Iwabuchi K, Amemiya A, et al: Immunohistochemical analysis of a case of gastritis cystica profunda associated with carcinoma development. Scand J Gastroenterol 1998;33:1226-1229.

Fonde EC, Rodning CB: Gastritis cystica profunda. Am J Gastroenterol 1986;81:459-464.

Kondo K: Duodenogastric reflux and gastric stump carcinoma. Gastric Cancer 2002;5:16-22.

Moon SY, Kim KO, Park SH, et al: Gastritis cystica profunda accompanied by multiple early gastric cancers. Korean J Gastroenterol 2010;55:325-330.

-5 Okada M, Iizuka Y, Oh K, et al: Gastritis cystica profunda presenting as giant gastric mucosal folds: the role of endoscopic ultrasonography and mucosectomy in the diagnostic work-up. Gastrointest Endosc 1994;40: 640-644.

6 Iwanaga T, Koyama H, Takahashi Y, et al: Diffuse submucosal cysts and carcinoma of the stomach. Cancer 1975;36:606-614.

7 Yamagiwa H, Matsuzaki O, Ishihara A, et al: Heterotopic gastric glands in the submucosa of the stomach. Acta Pathol Jpn 1979;29:347-350.

8 Littler ER, Gleibermann E: Gastritis cystica polyposa (gastric mucosal prolapse at gastroenterostomy site, with cystic and infiltrative epithelial hyperplasia). Cancer 1972;29:205-209.

-9 Franzin G, Novelli P: Gastritis cystica profunda. Histopathology 1981;5:535-547.

10 Bechade D, Desrame J, Algayres JP: Gastritis cystica profunda in a patient with no history of gastric surgery. Endoscopy 2007;39(suppl 1):E80-E81.

11 Park JS, Myung SJ, Jung HY, et al: Endoscopic treatment of gastritis cystica polyposa found in an unoperated stomach. Gastrointest Endosc 2001;54:101-103.

12 Wang TC, Dangler CA, Chen D, et al: Synergistic interaction between hypergastrinemia and Helicobacter infection in a mouse model of gastric cancer. Gastroenterology 2000;118:36-47.

$\$ 13$ Hedenbro JL, Ekelund M, Wetterberg P: Endoscopic diagnosis of submucosal gastric lesions. The results after routine endoscopy. Surg Endosc 1991;5:20-23.

-14 Koga S, Watanabe H, Enjoji M: Stomal polypoid hypertrophic gastritis: a polypoid gastric lesion at gastroenterostomy site. Cancer 1979;43:647-657. 


\section{Case Reports in \\ Gastroenterology}

\begin{tabular}{l|l}
\hline Case Rep Gastroenterol 2014;8:270-275 \\
\hline DOI: 10.1159/000368076 & $\begin{array}{l}\text { ○ 2014 S. Karger AG, Basel } \\
\text { www.karger.com/crg }\end{array}$ \\
\hline
\end{tabular}

Ogasawara et al.: A Case of Early Gastric Cancer Arising from Gastritis Cystica Profunda Treated by Endoscopic Submucosal Dissection
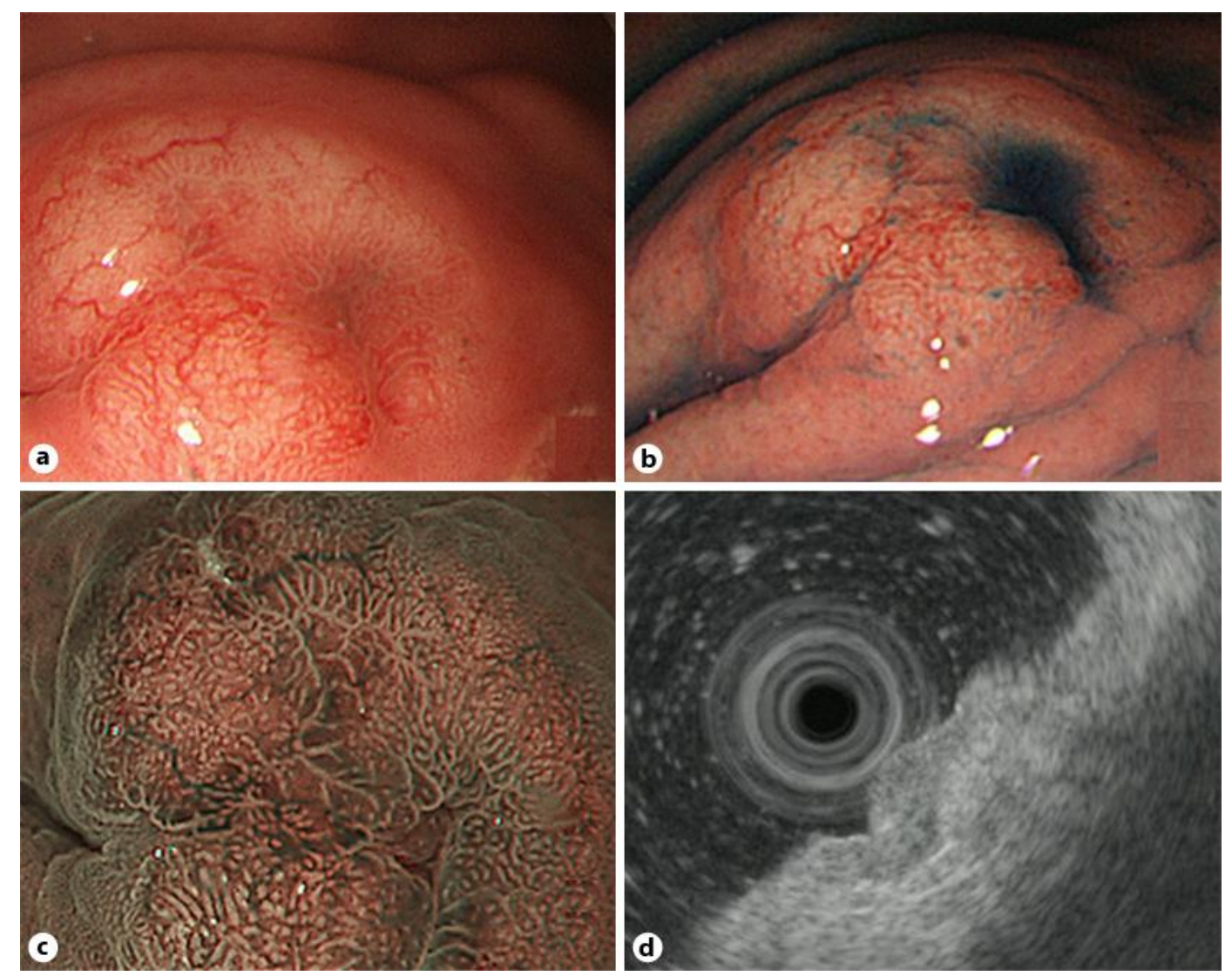

Fig. 1. a, b Endoscopic view showing the 15-mm diameter SMT with normal overlying mucosa in the giant curvature of the upper corpus of the stomach (a). The surface of the SMT had angiotelectasia (a) and slight depression covered with normal mucosa, stained with indigo carmine dye (b). However, neither ulceration nor erosion was seen $(\mathbf{a}, \mathbf{b})$. c Narrow-band imaging endoscopy showed no abnormal finding suggesting gastric cancer. The mucosa of the whole stomach was normal without chronic gastritis. d EUS visualized the SMT mainly within the second and third layers of the gastric wall. The tumor was internally low-echoic and harbored tiny cystic lesions. 

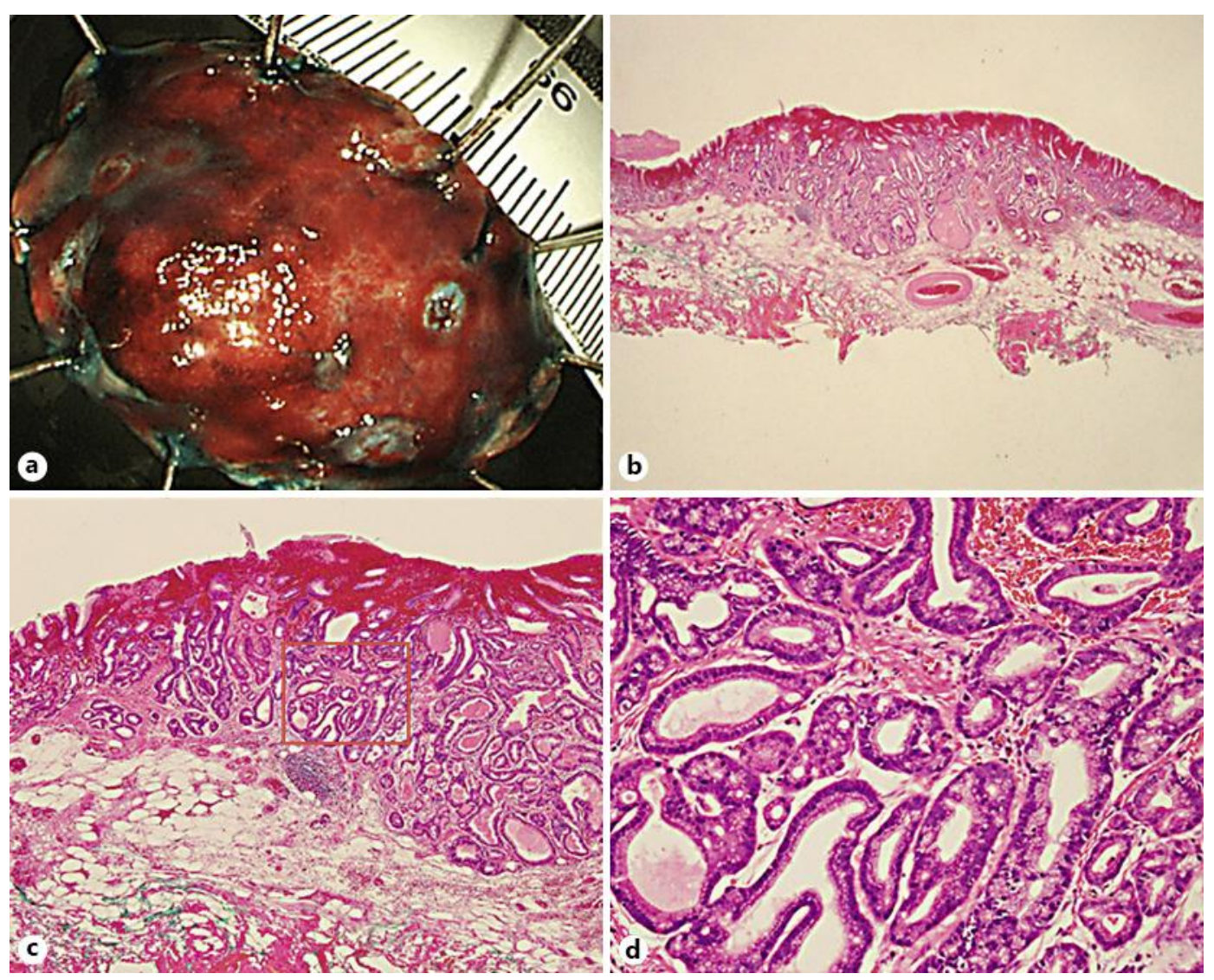

Fig. 2. Pathological findings of the resected SMT. a Complete SMT resection was confirmed. The resected specimen was $35 \times 20 \mathrm{~mm}$ and the tumor was $12 \times 12 \mathrm{~mm}$. b, c Cystic dilatation of the gastric glands extending into the submucosal layer was present, and the SMT was diagnosed as GCP. The gastric epithelium comprised normal mucosa without any dysplasia (c). $\mathbf{d}$ Inset of $\mathbf{c}$. Well-differentiated adenocarcinoma was partially seen within the GCP. There was no involvement of the lateral and deep margins, lymphatic invasion, vascular invasion or perineural invasion. Original magnification: $\times 10$ (b), $\times 100$ (c), $\times 400$ (d). 